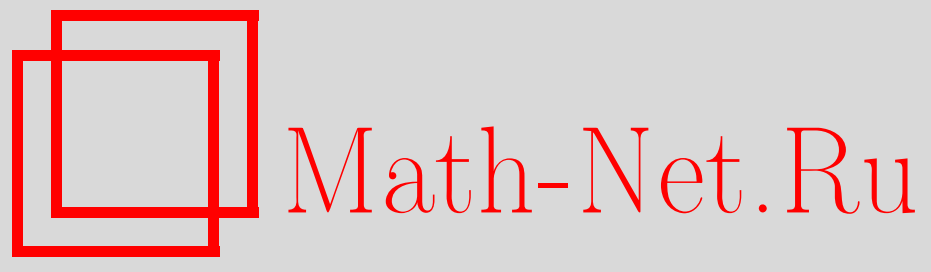

В. К. Леонтьев, О корнях случайных полиномов над конечным полем, Матем. заметки, 2006, том 80, выпуск 2, 313-316

DOI: https://doi.org/10.4213/mzm2812

Использование Общероссийского математического портала Math-Net.Ru подразумевает, что вы прочитали и согласны с пользовательским соглашением http://www . mathnet.ru/rus/agreement

Параметры загрузки:

IP : 54.209 .52 .79

26 апреля 2023 г., 16:12:33

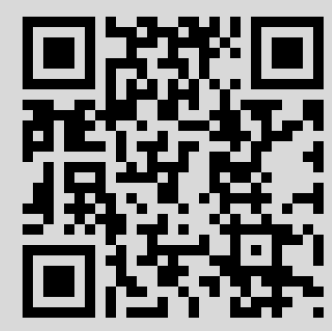




\section{О КОРНЯХ СЛУЧАЙНЫХ ПОЛИНОМОВ НАД КОНЕЧНЫМ ПОЛЕМ}

\section{В. К. Леонтьев}

Пусть $F_{q}$ - конечное поле и $F_{q}[x]$ - кольцо полиномов над полем $F_{q}$. Для полинома $f(x) \in F_{q}[x]$ мы обозначим через $\lambda(f)$ число корней $f(x)$, лежащих в поле $F_{q}$. В статье обсуждается проблема статистических свойств величины $\lambda(f)$ при равномерном распределении на множестве нормированных полиномов ${ }^{1}$ степени не выше, чем $m$. Такого рода проблемы встречаются при изучении перестановочных полиномов и в ряде разделов теории корректирующих кодов [1]-[3]. Для вещественного поля $\mathbb{R}$ теорема М. Каца утверждает, что "в среднем" полином степени $n$ с вещественными коэффициентами, нормально и независимо распределенными на отрезке $[0,1]$, имеет асимптотически $\left(\log _{2} n\right) /(2 \pi)$ вещественных корней [4].

Основной результат настоящей работы состоит в следующем: число нулей случайного полинома из $F_{q}[x]$, лежащих внутри поля $F_{q}$, имеет в асимптотике $(q \rightarrow \infty)$ распределение Пуассона с параметром $\lambda=1$. В частности, случайный полином из $F_{q}[x]$ при растущем $q$ имеет "в среднем" ровно один корень в поле $F_{q}$.

Пусть $t_{m}$ - число нормированных (с коэффициентом при старшей степени, равным единице) полиномов степени $m$, не имеющих корней в поле $F_{q}$ (здесь $q=p^{r}$ и $p$ - простое число).

Лемма 1. Имеет место равенство

$$
t_{m}=\sum_{i=0}^{m}(-1)^{i}\left(\begin{array}{l}
q \\
i
\end{array}\right) q^{m-i} .
$$

ДокАзАтЕЛЬСтво. Обозначим через $Z_{m}^{i}$ множество полиномов степени $m$ из $F_{q}[x]$, имеющих корень $\alpha_{i} \in F_{q}=\left\{\alpha_{1}=0, \alpha_{2}, \ldots, \alpha_{q}\right\}$, и пусть $Z_{m}=\bigcup_{i=1}^{q} Z_{m}^{i}$. Тогда

$$
t_{m}=q^{m}-\left|Z_{m}\right|
$$

где $\left|Z_{m}\right|$ - мощность множества $Z_{m}$. Применяя формулу “включений-исключений”, из (2) получаем

$$
\left|Z_{m}\right|=\sum_{s=1}^{m}(-1)^{s-1} \sum_{1 \leqslant i_{1} \leqslant \cdots \leqslant i_{s} \leqslant q}\left|Z_{m}^{i_{1}} \cap Z_{m}^{i_{2}} \cap \cdots \cap Z_{m}^{i_{s}}\right| .
$$

Если нормированный полином $f(x) \in F_{q}[x]$ степени $m$ имеет в качестве корней элементы $\alpha_{i_{1}}, \alpha_{i_{2}}, \ldots, \alpha_{i_{s}}$ из поля $F_{q}$, то

$$
f(x)=\left(x-\alpha_{i_{1}}\right) \ldots\left(x-\alpha_{i_{s}}\right) \varphi(x),
$$

где $\varphi(x)$ - произвольный нормированный полином степени $m-s$ из $F_{q}[x]$. Отсюда следует, что

$$
\left|Z_{m}^{i_{1}} \cap Z_{m}^{i_{2}} \cap \cdots \cap Z_{m}^{i_{s}}\right|=q^{m-s} .
$$

Из (2)-(4) следует (1).

Применяя лемму, вычислим для примера значения $t_{m}$ при $m=0,1,2,3: t_{0}=1, t_{1}=0$, $t_{2}=\left(\begin{array}{c}q \\ 2\end{array}\right), t_{3}=q\left(q^{2}-1\right) / 3$.

Работа выполнена при финансовой поддержке Российского фонда фундаментальных исследований, проект 05-01-01019.

${ }^{1}$ Всюду ниже, если специально не оговаривается, речь идет только о нормированных полиномах из $F_{q}[x]$.

(C) B. К. ЛЕонтьев, 2006 
СледствиЕ 1. Пусть $B_{m, q, r}$ - число полиномов степени $m$ из $F_{q}[x]$, имеющих ровно $r$ различных корней в поле $F_{q}$. Тогда

$$
B_{m, q, r}=\left(\begin{array}{c}
q \\
r
\end{array}\right) \sum_{i=0}^{m-r}\left(\begin{array}{c}
r+i-1 \\
r-1
\end{array}\right) t_{m-(r+i)}
$$

ДокАзАтЕЛЬСтво. Если полином $f(x)$ имеет $r$ различных корней в поле $F_{q}$, то

$$
f(x)=\left(x-\alpha_{1}\right)^{\lambda_{1}}\left(x-\alpha_{2}\right)^{\lambda_{2}} \ldots\left(x-\alpha_{r}\right)^{\lambda_{r}} \varphi(x),
$$

где все $\alpha_{i}$ различны, $\lambda_{i} \geqslant 1$ и $\varphi(x)$ не имеет корней в $F_{q}$. Отсюда следует соотношение

$$
B_{m, q, r}=\left(\begin{array}{l}
q \\
r
\end{array}\right) \sum_{k=r}^{m}\left(\begin{array}{l}
k-1 \\
r-1
\end{array}\right) t_{m-k}=\left(\begin{array}{l}
q \\
r
\end{array}\right) \sum_{i=0}^{m-r}\left(\begin{array}{c}
r+i-1 \\
r-1
\end{array}\right) t_{m-(r+i)}
$$

Пусть далее

$$
t_{m, \lambda}(q)=\sum_{i=0}^{m}(-1)^{i}\left(\begin{array}{c}
q \\
i
\end{array}\right) \lambda^{m-i}
$$

и $F(x)$ - производящая функция последовательности $\left\{t_{m, \lambda}(q)\right\}_{m=0}^{\infty}$, т.е.

$$
F(x)=\sum_{m=0}^{\infty} t_{m, \lambda}(q) x^{m} .
$$

Лемма 2. Имеет место равенство

$$
F(x)=\frac{(1-x)^{q}}{1-\lambda x} .
$$

Равенство (6) доказывается "прямым" вычислением.

Далее мы используем стандартный формализм из [5]:

$$
\begin{aligned}
\left(\begin{array}{c}
r+i-1 \\
i
\end{array}\right) & =\operatorname{Coef}_{u}\left\{\frac{1}{(1-u)^{r} u^{i+1}}\right\} \\
t_{m-(r+i)} & =\operatorname{Coef}_{u}\left\{\frac{(1-u)^{q}}{(1-u q) u^{m-r-i+1}}\right\}, \\
t_{m, \lambda}(q) & =\operatorname{Coef}_{u}\left\{\frac{(1-u)^{q}}{(1-\lambda u) u^{m+1}}\right\} .
\end{aligned}
$$

Лемма 3. Имеет место равенство

$$
B_{m, q, r}=\left(\begin{array}{l}
q \\
r
\end{array}\right) t_{m-r, q}(q-r) .
$$

ДоказАтЕЛЬСтво. Из (5) и (7) имеем

$$
\sum_{i=0}^{m-r}\left(\begin{array}{c}
r+i-1 \\
r-1
\end{array}\right) t_{m-(r+i)}=\sum_{i=0}^{\infty} \operatorname{Coef}_{u}\left\{\frac{1}{(1-u)^{i+1} u^{r}}\right\} \operatorname{Coef}_{v}\left\{\frac{(1-v)^{q}}{(1-v q) v^{m-r-i+1}}\right\} .
$$

Из (9) после рутинных вычислений получаем

$$
\begin{aligned}
\sum_{i=0}^{m-r}\left(\begin{array}{c}
r+i-1 \\
r-1
\end{array}\right) t_{m-(r+i)} & =\operatorname{Coef}_{v}\left\{\frac{(1-v)^{q}}{(1-v q) v^{m-r+1}} \operatorname{Coef}_{u}\left\{\frac{1}{u^{r}(1-u)} \cdot \frac{1}{1-\frac{v}{1-u}}\right\}\right\} \\
& =\operatorname{Coef}_{v}\left\{\frac{(1-v)^{q}}{(1-v q) v^{m-r+1}(1-v)^{r}}\right\}=t_{m-r, q}(q-r) .
\end{aligned}
$$


Пусть $N_{m}=\left\{f_{1}, f_{2}, \ldots, f_{q} m\right\}$ - множество всех полиномов степени $m$ из $F_{q}[x]$. Зададим на $N_{m}$ равномерное вероятностное распределение, приписав каждому полиному из $N_{m}$ вероятность $1 / q^{m}$. Рассмотрим случайную величину $\lambda(f)$ - число различных корней полинома $f$, лежащих в поле $F_{q}$, где $f$ - случайно выбранный полином из $N_{m}$. Рассмотрим распределение

$$
q_{r}^{(m)}=\mathrm{P}\{\lambda(f)=r\}, \quad r=0,1,2, \ldots,
$$

и производящую функцию этого распределения

$$
\Phi_{m}^{q}(x)=\sum_{r=0}^{\infty} q_{r}^{(m)} x^{r} .
$$

Лемма 4. Имеет место равенство

$$
\Phi_{m}^{q}(x)=\sum_{i=0}^{m} \frac{\left(\begin{array}{c}
q \\
i
\end{array}\right)}{q^{i}}(x-1)^{i} .
$$

ДокАЗАТЕЛЬСТво. Используя лемму 3, получаем

$$
\begin{aligned}
\Phi_{m}^{q}(x) & =\sum_{r=0}^{m} \frac{B_{m, q, r}}{q^{m}} x^{r}=\frac{1}{q^{m}} \sum_{r=0}^{m} x^{r}\left(\begin{array}{l}
q \\
r
\end{array}\right) t_{m-r, q}(q-r) \\
& =\frac{1}{q^{m}} \sum_{r=0}^{m} x^{r}\left(\begin{array}{l}
q \\
r
\end{array}\right) \operatorname{Coef}_{u}\left\{\frac{(1-u)^{q-r}}{(1-u q) u^{m-r+1}}\right\} \\
& =\frac{1}{q^{m}} \operatorname{Coef}_{u}\left\{\frac{(1-u)^{q}}{u^{m+1}(1-u q)} \sum_{r=0}^{\infty}\left(\frac{u x}{1-u}\right)^{r}\left(\begin{array}{l}
q \\
r
\end{array}\right)\right\}=\frac{1}{q^{m}} \operatorname{Coef}_{u}\left\{\frac{(1+u(x-1))^{q}}{(1-u q) u^{m+1}}\right\} \\
& =\frac{1}{q^{m}} \sum_{s=0}^{q}\left(\begin{array}{l}
q \\
s
\end{array}\right)(x-1)^{s} \operatorname{Coef}_{u}\left\{\frac{1}{u^{m-s+1}}\left[1+(u q)+(u q)^{2}+\cdots\right]\right\} \\
& =\frac{1}{q^{m}} \sum_{s=0}^{q}\left(\begin{array}{l}
q \\
s
\end{array}\right)(x-1)^{s} q^{m-s}=\sum_{s=0}^{m} \frac{\left(\begin{array}{c}
q \\
s
\end{array}\right)}{q^{s}}(x-1)^{s} .
\end{aligned}
$$

Во всех выкладках учитывается, что $r \leqslant \min \{q, m\}$.

Пусть теперь $\mathrm{M}(\lambda(f))$ и $\mathrm{D}(\lambda(f))$ - соответственно математическое ожидание и дисперсия случайной величины $\lambda(f)$.

Теорема 1. Имеют место равенства

$$
\mathrm{M}(\lambda(f))=1, \quad \mathrm{D}(\lambda(f))=1-\frac{1}{q} .
$$

Это утверждение сразу следует из леммы 4, так как первая и вторая производные функции $\Phi_{m}^{q}(x)$ легко вычисляются в точке $x=1$.

Пусть теперь $M_{n}=\{f\}-$ множество всех полиномов степени $\leqslant n$ из $F_{q}[x]$. На множестве $M_{n}$ зададим равномерное вероятностное распределение, приписав каждому полиному $f$ из $M_{n}$ вероятность $1 / N$, где $N=\left|M_{n}\right|=\left(q^{n+1}-1\right) /(q-1)$. Рассмотрим ту же самую случайную величину $\lambda_{n}(f)$ - число различных корней полинома $f$ из $M_{n}$, лежащих в поле $F_{q}$.

Теорема 2. Если $n=q-1 u q \rightarrow \infty$, то случайная величина $\lambda_{n}(f)$ имеет в пределе распределение Пуассона с параметром $\lambda=1$. 
ДокАЗАТЕльство. Используя лемму 3 и формулу полной вероятности, получаем

$$
\begin{aligned}
\mathrm{P}\left\{\lambda_{n}(f)=r\right\} & =\mathrm{P}_{r}=\frac{1}{N} \sum_{m=1}^{n} q^{m} q_{r}^{(m)}=\frac{\left(\begin{array}{c}
q \\
r
\end{array}\right)}{N} \sum_{m=1}^{n} \operatorname{Coef}_{u}\left\{\frac{(1-u)^{q-r}}{(1-q u) u^{m-r+1}}\right\} \\
& =\frac{\left(\begin{array}{c}
q \\
r
\end{array}\right)}{N} \operatorname{Coef}_{u}\left\{\frac{(1-u)^{q-r-1}}{(1-q u) u^{n-r+1}}\right\}=\frac{\left(\begin{array}{c}
q \\
r
\end{array}\right)}{N} t_{n-r, q}(q-r-1) .
\end{aligned}
$$

Используя (12), можно найти производящую функцию распределения $\left\{\mathrm{P}_{r}\right\}$ :

$$
\begin{aligned}
\Phi_{q}^{n}(x) & =\frac{1}{N} \sum_{r=0}^{\infty}\left(\begin{array}{l}
q \\
r
\end{array}\right) t_{n-r, q}(q-r-1) x^{r}=\frac{1}{N} \sum_{r=0}^{\infty}\left(\begin{array}{l}
q \\
r
\end{array}\right) x^{r} \underset{u}{\operatorname{Coef}}\left\{\frac{(1-u)^{q-r-1}}{(1-q u) u^{n-r+1}}\right\} \\
& =\frac{1}{N} \operatorname{Coef}_{u}\left\{\frac{(1-u)^{q-1}}{(1-q u) u^{n+1}} \sum_{r=0}^{q}\left(\begin{array}{c}
q \\
r
\end{array}\right)\left(\frac{x u}{1-u}\right)^{r}\right\}=\frac{1}{N} \underset{u}{\operatorname{Coef}}\left\{\frac{[1+u(x-1)]^{q}}{(1-q u)(1-u) u^{n+1}}\right\} .
\end{aligned}
$$

Отсюда

$$
\Phi_{q}^{n}(x)=\frac{1}{2 \pi i N} \oint_{|u|=\rho} \frac{[1+u(x-1)]^{q}}{(1-q u)(1-u) u^{n+1}} d u .
$$

Здесь $\rho$ - достаточно малое положительное число.

Функция $\Psi(u)$, заданная следующим образом:

$$
\Psi(u)=\frac{[1+u(x-1)]^{q}}{(1-u q)(1-u) u^{n+1}}
$$

имеет следующие особые точки: $u_{1}=0, u_{2}=q^{-1}, u_{3}=1, u_{4}=\infty$. Так как $n=q-1$, то

$$
\underset{u=u_{4}}{\operatorname{res}} \Psi(u)=0 .
$$

Далее,

$$
\underset{u=u_{2}}{\operatorname{res}} \Psi(u)=q^{n} \frac{(1+(x-1) / q)^{q}}{1 / q-1}, \quad \underset{u=u_{3}}{\operatorname{res}} \Psi(u)=\frac{x^{q}}{q-1} .
$$

Используя теперь теорему о вычетах, получаем

$$
\Phi_{q}^{n}(x)=\frac{q^{n+1}-q^{n}}{q^{n+1}-1}\left(1-\frac{x-1}{q}\right)^{q}-\frac{x^{q}}{q^{n+1}-1} .
$$

Из (14) следует

$$
\lim _{q \rightarrow \infty} \Phi_{q}^{n}(x)=e^{x-1}
$$

для произвольной точки $x$ из $(0,1]$. Утверждение теоремы 2 теперь следует из теоремы о непрерывности для производящих функции [6].

\section{СПИСОК ЦИТИРОВАННОЙ ЛИТЕРАТУРЫ}

[1] Р. Лидл, Г. Нидеррайт, Конечнъе поля, 1, 2, Мир, М., 1988. [2] Дж. Мак-Вильямс, Н. Дж. А. Слоэн, Теория кодов, исправляющих ошибки, Связь, М., 1979. [3] В. К. Леонтьев, Труды IV Межд. конф. "Дискретные модели в теории управляющих систем", 2000. [4] М. Кац, Вероятность и смежные вопросы в физике, Мир, М., 1965. [5] Г.П. Егорычев, Интегральное представление и вычисление комбинаторных сумм, Наука, Новосибирск, 1977. [6] В.Н. Сачков, Вероятностные методы в комбинаторном анализе, Наука, М., 1978.

\section{В. К. Леонтьев}

Вычислительный центр РАН 